\title{
Application of Wireless Sensor Network and RFID Monitoring System in Airport Logistics
}

\author{
https://doi.org/10.3991/ijoe.v14i01.8058 \\ Le Wang \\ Xi' an University of Architecture and Technology, Xi'an, Shaanxi, China \\ Dalian University, Dalian, Liaoning, China \\ hetekkb41966@yeah. net
}

\begin{abstract}
To better regulate the airport cargo transportation, we presented the design of an airport logistics monitoring system based on WSN and RFID. The monitoring system is mainly composed of four modules: RFID system, wireless sensor monitoring network, communication network and remote monitoring terminal. Wireless sensors mainly include temperature sensors, humidity sensors and smoke sensors. The sensors can collect the environment data such as goods temperature, humidity and smoke and so on. The node sensor module is implemented in such a way that the sensor is connected to the node in a plugin manner, that is, the sensors are integrated on a sensor panel and the sensor data is transferred through the standard I / O interface between the sensor panel and the node. The system can realize real-time tracking and positioning of airport cargo, collecting cargo information.
\end{abstract}

Keywords-logistics; wireless sensor network; ZigBee; GPRS; remote monitoring

\section{Introduction}

With the continuous development of air transport industry in recent years, the airport air cargo volume is also showing a rapid growth trend, so efficient storage and management of a large number of airport cargo has become a very important issue. Many large international airports cover an area of tens of square kilometers, distributing thousands of luggage cabinets belonging to different airlines, as well as a large number of carts and transport vehicles. For such a large scale and a variety of objects, simply relying on the human resource management will inevitably lead to leaks, goods or means of transportation lost or misplaced event, affecting the normal operation of the whole system. As a result, the airport must be equipped with efficient logistics management system to implement the effective management of these objects, so as to ensure the smooth and orderly operation. 


\section{State of art}

In recent years, wireless sensor network has become the concern of the domestic and foreign new hot research field. It is composed of multi inter-disciplines, and through various integrated micro sensor nodes coordination, it completes monitoring, sensing and collecting all kinds of environmental information or object monitoring information, and makes information processing. Through random self organization wireless communication network with multi hop relay, the sensed information will be transmitted to the user terminal. The wireless sensor network has the advantages of small size sensor nodes, low price, small power consumption, high precision, large coverage, remote monitoring and many other advantages, and the network has better robustness and invulnerability [1].

RFID is a radio frequency tag, which uses wireless automatic identification technology. It is a small transmitting device that can encode and read items by wireless tags. In RFID tags, the specified and interoperable information are stored, and through a wireless data communication network, they are automatically collected to a central information system. The purpose is to realize the identification of the goods, and then through the open computer network, to realize information exchange and sharing. RFID application system consists of three parts: RFID tag, RFID reader and RFID data management system. Each tag has the world's unique identification number (ID), cannot be modified or copied, with high security [2].

But the anti-interference of RFID is relatively poor, and the effective distance is generally less than $10 \mathrm{~m}$, while the communication coverage of wireless sensor network is tens of meters. If combined wireless sensor with RFID, it will expand its scope of application. Thus, it can easily find the node location by RFID, and can make the network focused on the acquisition of environmental data [3]. The advantages of RFID and wireless sensor network technology in airport logistics mainly lie in the sorting of luggage goods, real-time tracking of goods and warehousing management of goods.

Airport logistics mainly includes three parts: cargo handling, baggage handling and catering handling. This paper is mainly for cargo handling in logistics. Through the use of active RFID tags, RFID reader and the wireless sensor network, the airport can establish a new procedure to automatically track all air freights, so as to ensure safe transportation and flow of millions of goods. The process of goods entering and leaving the port is shown in Figure 1 [4].

For instance, when cargo leaves the port, after receiving the Airlines cargo information, the airport will prepare the yard unloading spaces in advance, which is based on the name of goods, category, flights, routes, the number of trucks and other information. After trucks arrived at the airport, they entered the parking lot, waiting for a dump truck. Airport control center, according to the relevant information of goods, flights and unloading parking spaces queuing, reasonably schedule the unloading parking spaces, to ensure that goods can be timely loaded and unloaded. Air cargo, in the airport cargo station after security check into the storage, is ready to board the departure port. The operation of this process can be realized by RFID technology, and according to the read goods electronic tag data information, related work arrange- 


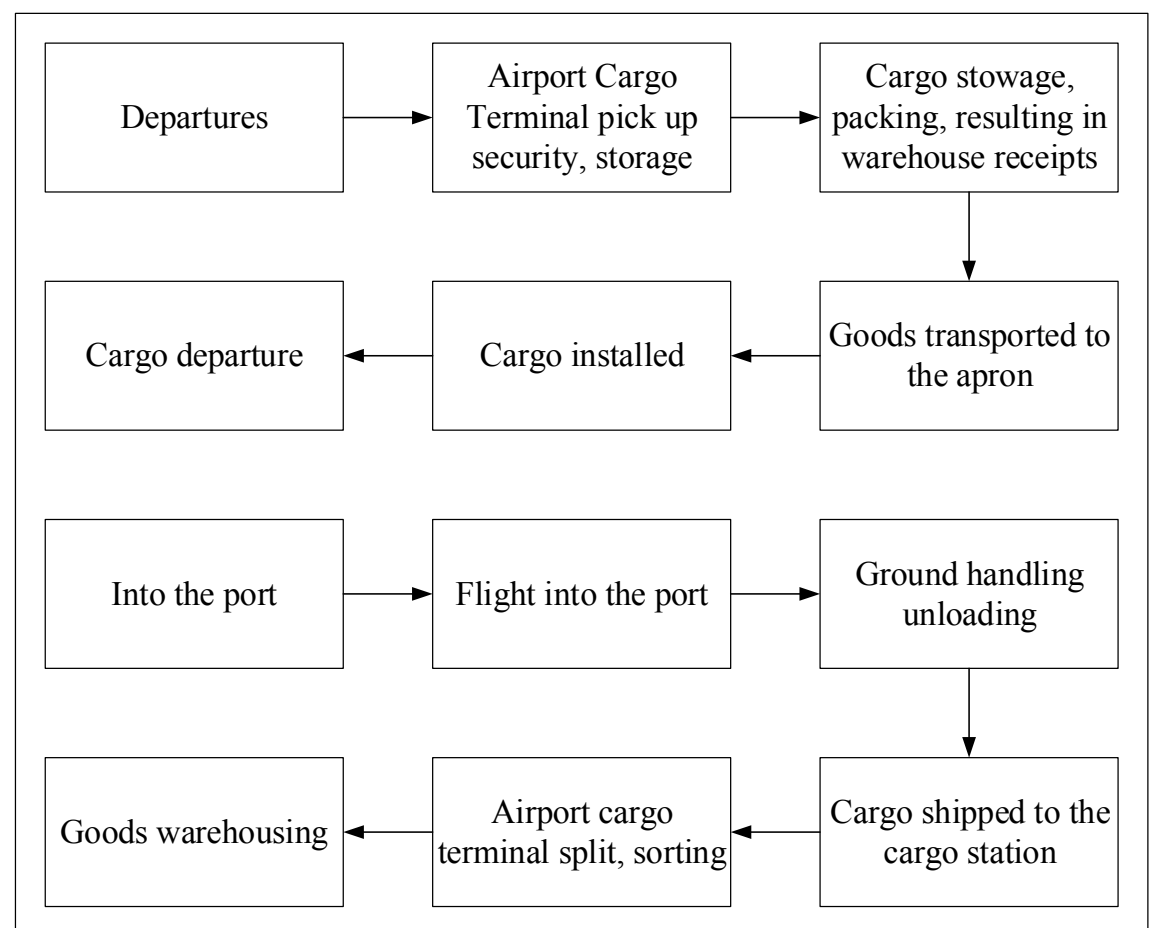

Fig. 1. Remote monitoring system structure

ments and scheduling are made. In addition, the wireless sensor network technology can realize the real-time monitoring of the environment in the process of air cargo transportation, such as temperature, humidity and smoke degree, so as to ensure that some special goods can arrive at the destination in good condition.

Therefore, this paper proposes a monitoring system based on wireless sensor network and RFID, to be applied to the airport logistics, so as to achieve the collection and real-time tracking of airport cargo related information, and realize real-time remote control. In this way, the flow of goods can be effectively supervised, and it provides great convenience for the scheduling of the airport control center, reduces the work burden of airport staffs, and greatly improves the management level of the airport logistics.

\section{System overall design}

According to the factors of airport logistics industry application characteristics and service needs, based on the networking and intelligent node network system, we design a three layer logistics monitoring system architecture including sensing layer, control layer and service layer. The specific implementation is shown below, as shown in figure 2. 


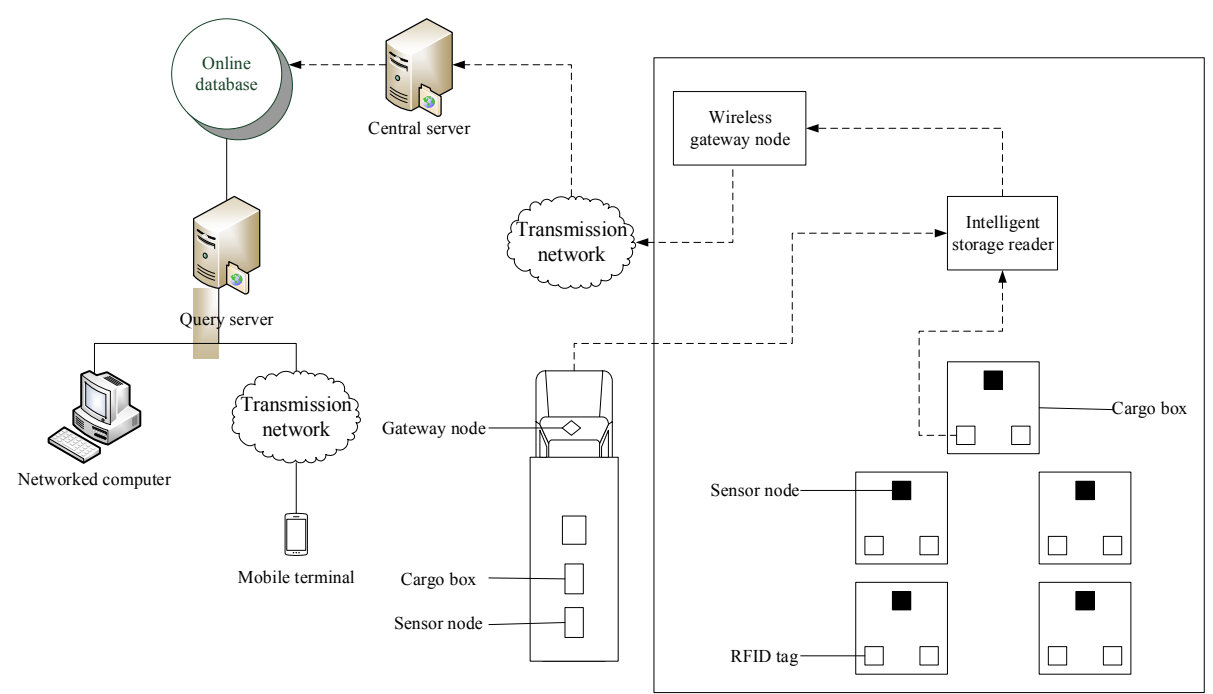

Fig. 2. Integration of WSN and RFID modern logistics monitoring system structure

In the above figure, the sensor layer consists of WSN sensor nodes, intelligent storage reader, RFID tag and wireless gateway node self organizing wireless data acquisition network. Wireless data acquisition network, with the intelligent storage reader as a network node, effectively integrates RFID technology and WSN technology together, which completes the network control and information gathering of each node. The sensing layer of the monitoring system is responsible for, in the logistics distribution center, delivery carriage or other application scenarios, sensing the environment parameters of goods, goods identification, quality characteristics and so on information. The wireless gateway node collects the information collected by other nodes, and sends to the service center of system control layer through the corresponding transmission network. Intelligent reader of sensor level of logistics monitoring system includes two kinds of fixed and mobile: the former can be placed into the warehouse entrance and goods shelves. Many readers will divide the warehouse into several monitoring areas and organize it into the network in advance; for mobile reader, it is mainly to facilitate the warehouse goods post sorting operation, and through the self organization ability of WSN, it also can facilitate the participation in the network.

The control layer of the system is composed of central server and online database, which is the link between wireless data acquisition network and enterprise applications. A large number of raw data from wireless gateway nodes can be of practical value to users only be screened, filtered and analyzed. After the processed data are stored to the online database, the center server complete the corresponding fitness of information according to the different needs of users, and transmit the note of relevant information according to the user setting; at the same time, in the working case of needing to control the WSN node and the RFID tag, the control layer will complete the generation of control instructions and the issued operation. 
The user layer of logistics monitoring system is also called for the service layer, to provide public information services and enterprise services, including network computer, hand-held terminal and other user equipment and client management software.

\subsection{Functional module division}

According to the above system framework, the module design method is used to divide the whole logistics warehousing monitoring management system, and the specific design is illustrated in figure 3.

As can be seen from the figure above, the whole logistics warehouse monitoring management system is divided into four subsystems: business management subsystem, equipment management subsystem, cargo positioning tracking subsystem and information support subsystem. Among them, warehousing management, outbound management, inventory alarm management, warehouse allocation and statistics management module compose the business management subsystem, to realize the key functions of warehouse management. The equipment management subsystem mainly realizes the monitoring and management of the intelligent reader, WSN node and RFID tags working status. The locating and tracking subsystem includes the positioning of warehouse staffs, goods position tracking and so on functions, which is a characteristic module of the whole warehouse monitoring and management system. The

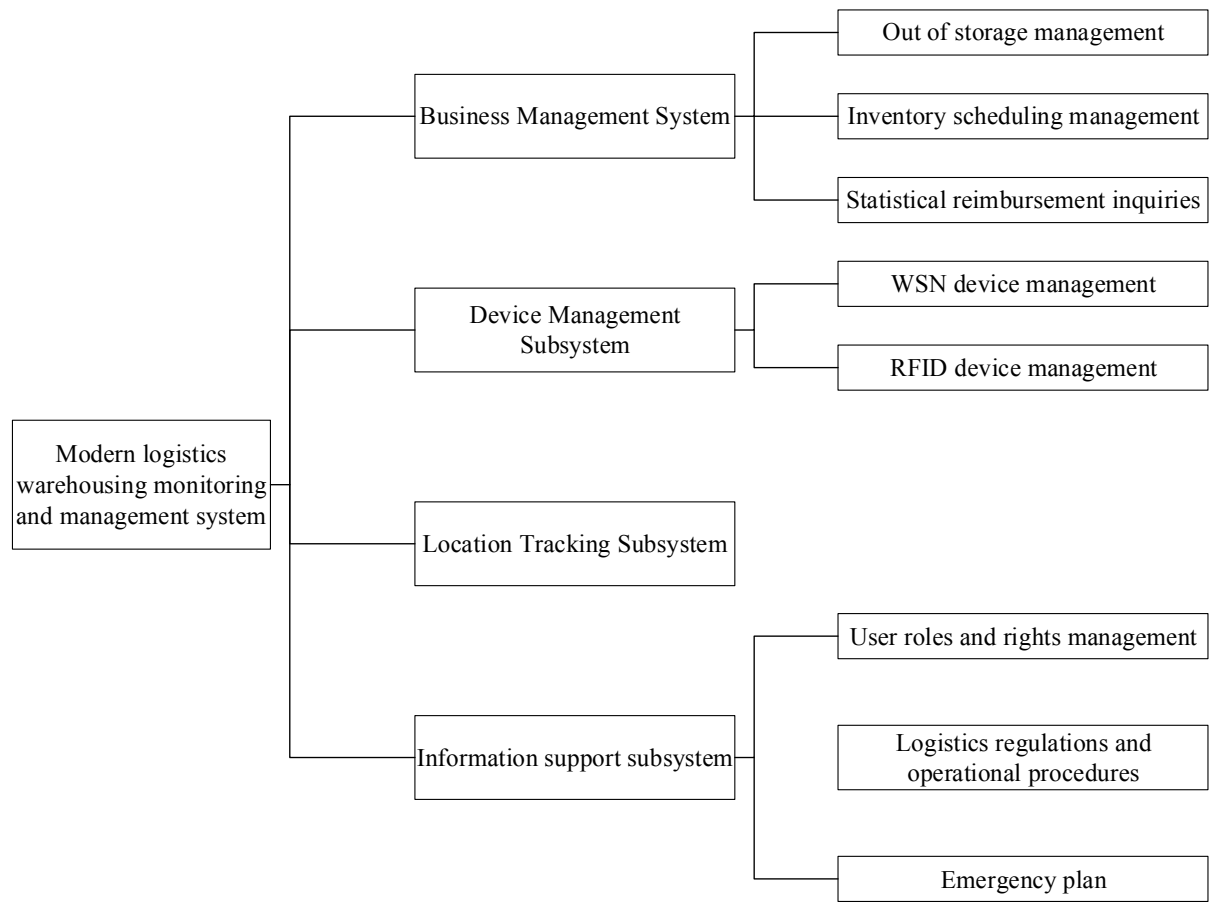

Fig. 3. Wireless sensor network sensor node structure 
information support subsystem is to provide decision support for the configuration and optimization of the system, composed of user authority management, laws and regulations of logistics, logistics processes and emergency contingency plans for handling and so on modules.

\subsection{Business process design}

Outbound and warehouse management: In the operation of logistics, the warehousing requires the establishment of warehousing rules, the identification of goods information, the verification of warehousing conditions, the production of inventory, cargo acceptance and storage and other operations, and its specific operation process is shown in figure 4.

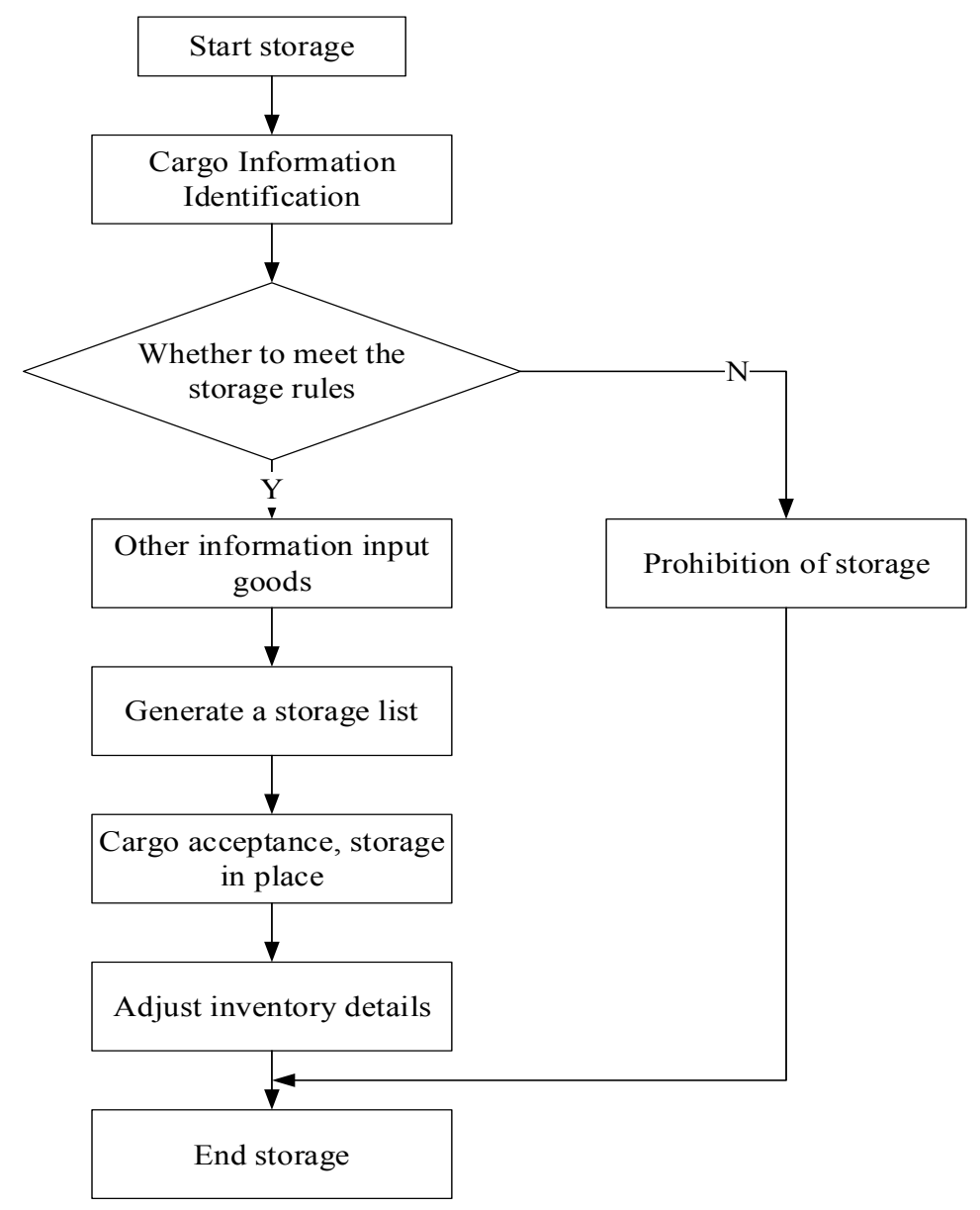

Fig. 4. Sink node power supply module circuit schematics 
In the above warehousing process, goods identification and access to information are implemented by the intelligent reader of the sensing layer. When the data is transmitted to the center server of the control layer, judge whether it meets the storage conditions. If the goods information meets the warehouse entry rules, the center server, for the application layer storage management software, issues instructions of goods, , the amount and other data, and generate ca detailed list of goods. And then according to the order, make the library storage, and finally, after the adjustment of inventory list, it completes the whole warehousing business process.

The outbound operation of goods can be viewed as the inverse process of warehousing operation, which needs application for goods out of warehouse, inventory check, generated detailed list of outbound goods, cargo extraction, update inventory list and other operations. The process is shown in figure 5.

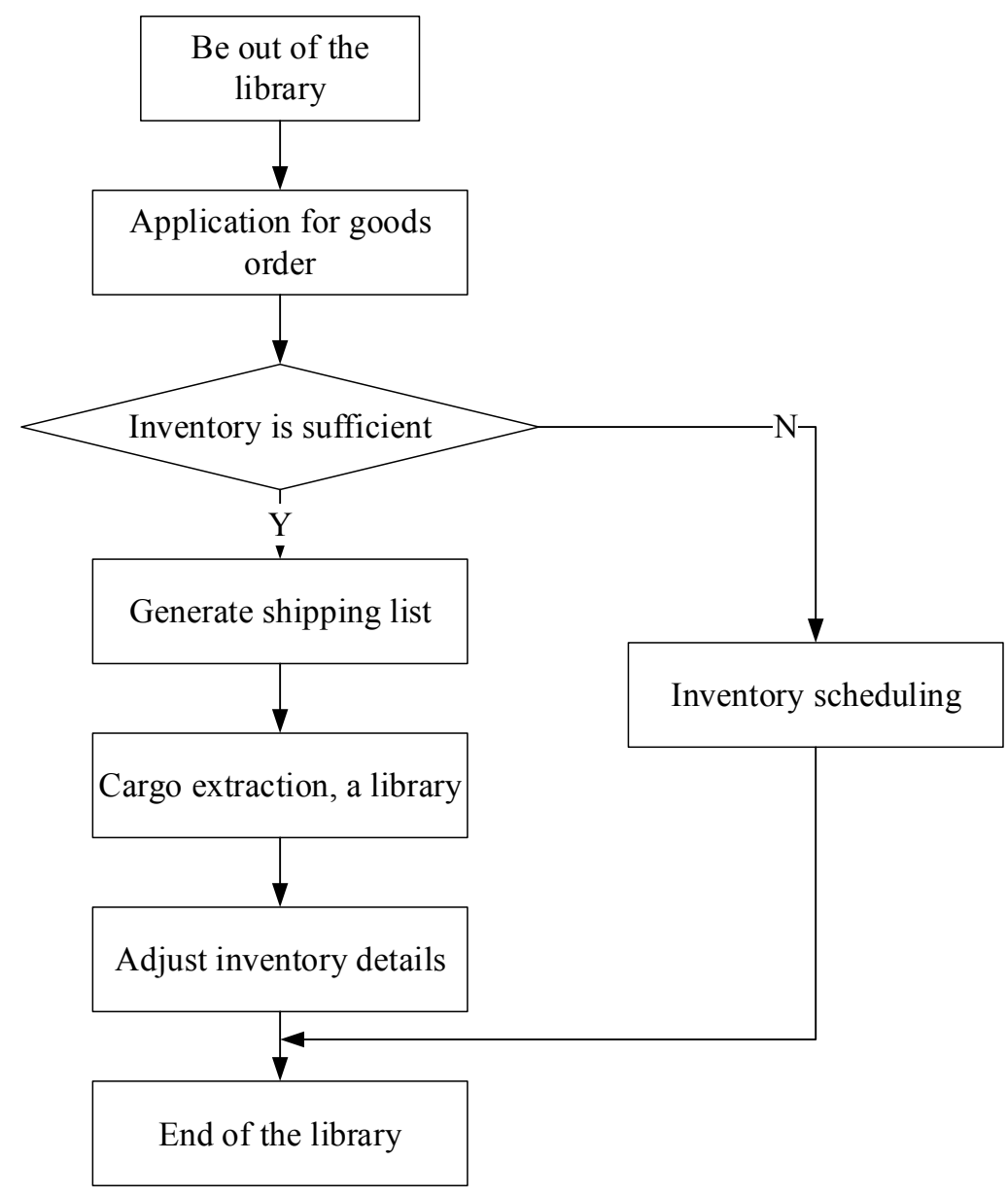

Fig. 5. Goods out of the warehouse management process 
After the sale of goods, according to the customer order or outbound application, warehouse manager, after ensure that the amount of inventory is sufficient, produce a detailed outbound list (if the inventory number is insufficient, we can apply for inventory scheduling). After the extraction of goods, we update inventory details, and complete the whole process of goods out of warehouse.

Inventory scheduling: Managed inventory management includes the adjustment of goods between different warehouses, as well as the quantity matching between the different shelves of the same warehouse when the goods are out of the warehouse. The former is the management method for goods out of warehouse when the total amount of goods is insufficient. The latter occurs in the case of sufficient total amount of goods in the same warehouse but in different shelves. For the latter, we use the principle that the goods that accounting the small proportion in the shelf is out of the warehouse firstly, to generate the list for goods out of the warehouse. For instance, the order requests for 15 computers out of warehouse, the shelf $\mathrm{A} 1$ has a total of 13 computers, A3 shelf has existing 10 computers, B2 and B3 have 5 computers each, C1 shelf has 3 computers, and $\mathrm{C} 2$ shelf has 2 computers, then system generates the list: 2 computers of $\mathrm{C} 2,3$ computers of $\mathrm{C} 1,5$ computers of $\mathrm{B} 3$, and 5 computers of B2. This principle can ensure that the warehouse has enough spare shelves, to facilitate other goods warehousing shelves.

Statistical query management: The warehouse should make statistical inventory regularly to ensure accurate warehousing data and timely inventory scheduling. The warehouse manager inquires the stock information through the intelligent reader, compares the newly obtained inventory information with the original inventory information, and carries out the corresponding operation according to the difference information of the stock.

In order to ensure the normal operation of goods logistics, the number of safety stock goods in the database in warehouse management monitoring system should be set; when the minimum amount of safety stock inventory query to a goods in stock is lower than the preset value, the system will trigger timely inventory control events, to remind the managers to make relevant decisions. The minimum safe inventory is determined by the order decision model in economics. The system designed in this paper uses the Economic Order Quantity (EOQ) model in the fixed order quantity model to determine the minimum safe inventory, which is described as follows:

$$
\begin{aligned}
& Q=M * D \\
& M=S * n
\end{aligned}
$$

In the above formulas, $\mathrm{Q}$ refers to the minimum quantity of inventory of warehouse, $\mathrm{M}$ indicates the average daily out of warehouse amount, D suggests the time of arrival of goods orders in this time period, $\mathrm{S}$ is the total outgoing amount of the goods for the latest $\mathrm{n}$ days, and $\mathrm{n}$ is set up by warehouse keeper in warehouse management system initialization. The model can minimize the sum of order cost and storage cost of logistics supply.

Equipment warehouse: The warehouse management is a complicated and meticulous work, a large number of inbound and outbound, inventory query, scheduling and 
other operations may make the system sensing layer equipment abnormal. Especially the WSN clustering nodes in the monitoring area, when dealing with a large number of goods information and environment parameters and so on data, will greatly consume energy. The equipment management module of the system can detect the working state of the intelligent reader and the WSN node, and eliminate the devices with abnormal working situation in the monitoring area. In order to reduce the energy loss of the system, we set up a reasonable interval reader to query the data; at the same time, we set turns work cycle for the WSN node, to maximize the network life cycle. Due to the limited radius of WSN single node RSSI (Received Signal Strength Indicator), the whole monitoring network may have communication dead corner. At this point, the cargo information of the communication dead corner area will be omitted in the inventory query statistics. Therefore, in the late period of the storage management, goods sorting and statistical query need warehouse managers to carry mobile readers to collect cargo information. In this way, the equipment management module can make network access authorization for mobile readers.

Positioning and tracking: In some cases, the operator takes a mobile reader to read information from different locations in the reservoir area. The monitoring system needs to know the location of these readers (operators) and record the start and end time of the operation. The positioning and tracking subsystem realizes the above functional requirements, and it is an innovation point of the whole warehouse monitoring management system. The system can monitor the location of the goods or the track of the personnel in the warehouse according to the ZigBee network composed of WSN nodes. The beacon nodes (reference nodes) location of positioning and tracking subsystem formed different monitoring regions around the layout area. When the mobile node (hand-held reader or mobile container) enters into a monitoring area, the beacon node can provide coordinate data, RSSI value and so on information for mobile nodes. Therefore, the mobile node can calculate the coordinate according to the data obtained from the beacon nodes. The positioning principle is shown in figure 6 .

The beacon node of the system uses the extended board with CC2430 chip, and the main chip of the mobile node adopts the CC2431 chip system with hardware positioning engine. When the CC2431 positioning engine calculates the coordinates of unknown nodes, it requires the system to provide the coordinates and RSSI values of 3 to 8 reference points. At the same time, it also needs to input the referred A value and $\mathrm{N}$ value. Among them, $\mathrm{A}$ is the RSSI value of 1 meters away from the transmitter, and the unit is dbm; $\mathrm{N}$ value represents the RSSI value of the distance transmitter every 1 meters attenuation, which is the environmental factor variable. When the CC2431 node collects the information needed in the network, the positioning engine is opened by the positioning control command, and the position coordinates of the positioning node are calculated. 


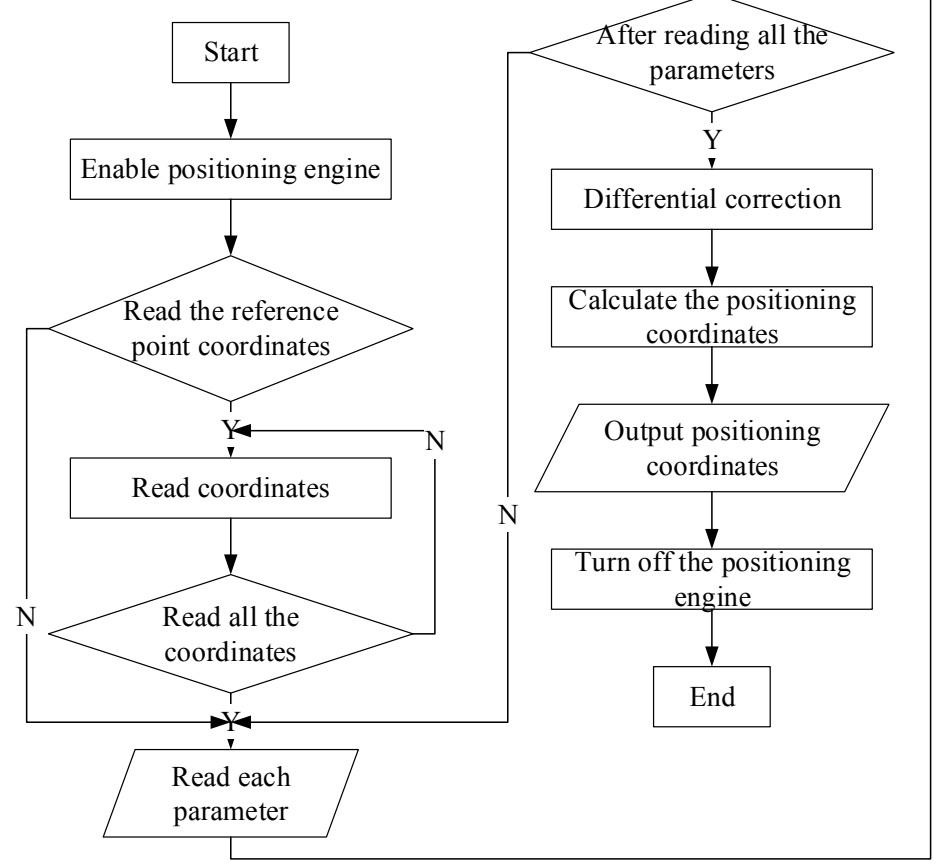

Fig. 6. Positioning tracking flow chart

\section{$4 \quad$ Key function design of logistics monitoring system integrating WSN and RFID}

According to modern logistics monitoring system overall design scheme for the integration of WSN and RFID, the design of key function of the system includes the following four parts: the design of intelligent reader of sensing layer; design of WSN and RFID data integration model of control layer; design of data intelligent storage process based on rules; design of logistics warehouse management and monitoring platform based on B/S (Browser/Server) mode of application layer.

\subsection{Smart reader design}

The hardware structure of smart reader is composed of microprocessor, RFID module and WSN module. AT89S52 single chip processor is connected to a temperature and humidity sensor, which is responsible for processing the data collected by the sensor, and controlling the RF module and the RFID module through the serial port RS232. Because the micro-controller cannot process the RS232 signal, the reader uses MAX232 to convert the TTL signal and the RS232 signal. The RF module is composed of a RF chip and antenna. The RF chip of the system uses the Chipcon compa- 
ny's CC2430, and reduces Zigbee protocol in the design to reduce the energy loss in the data transfer. The RFID module uses MFRC500 base station chip of PHILPS's MIFARE technology [5], which can meet the ISO/IEC14443A standard. In the meanwhile, it integrates encoding, modulation, anti-collision, security authentication and so on RFID control method and protocol, which can guarantee the stability of the RFID read and write operations. The hardware implementation is shown in figure 7.

The reader and WSN nodes constitute a new network, the intelligent reader xan read the dual information of the WSN node and the RFID tag, and it can communicate with the sensor nodes. It can also receive the data information sent by RFID tags and WSN sensor nodes and transmit it to the center server. While it uses intelligent reader as a WSN node energy provider to make most of the network management and information processing capabilities collected on intelligent reader device, which can fundamentally solve the energy consumption problem of WSN.

\subsection{Sensor node design}

The sensor node is a miniature embedded system, capable of sensing the physical environment data. Its processing capacity, storage capacity and communication capacity are relatively weak, which is powered by energy limited battery. From the network function, each sensor node considers both traditional network node terminal and router functions. In addition to local information collection and data processing, it is also necessary for the storage, management and integration processing of data sent by other nodes. At the same time, cooperating with other nodes, it completes some specific tasks. The sensor node is the focus of the wireless sensor network system, which is composed of a sensor module, processor module, wireless communication module and power supply module four parts, as shown in figure 8 .

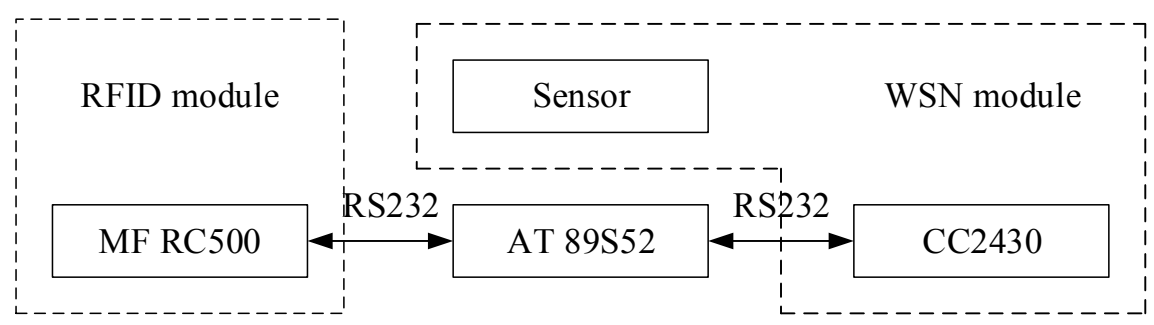

Fig. 7. Intelligent reader hardware structure diagram

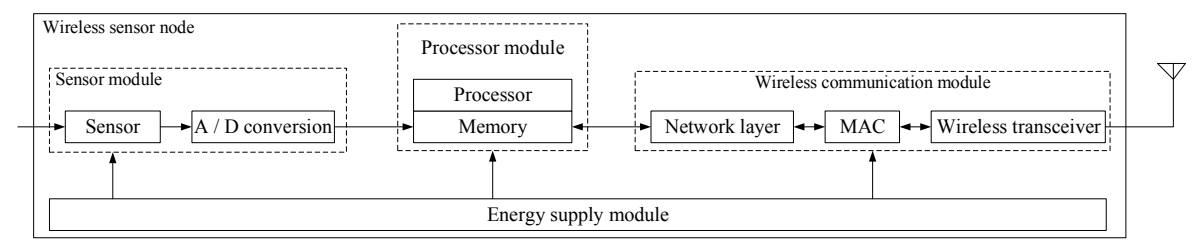

Fig. 8. Wireless sensor node structure 
The sensor module is responsible for monitoring the information collection and data conversion in the area. The sensor converts the measured physical quantity or signal into the corresponding analog signals such as voltage or current according to certain rules, and then the analog signals produced by the sensor are converted into digital signals through the $\mathrm{A} / \mathrm{D}$ conversion circuit.

The system mainly includes temperature sensor, humidity sensor and smoke sensor, which can collect the temperature, humidity and smoke degree of the environment where the goods are located. The realization mode of node sensor module is that the sensor is connected with the node in the form of plug-in. That is to say, these sensors are integrated on a sensing panel, and the sensing data transmission is realized through the standard I/O interface between the sensing panel and the node. The temperature sensor and humidity sensor selected DS18B20 and HS1101, respectively. DS18B20 is a new type of digital temperature sensor. When data is transmitted, it occupies only one I/O data line [7]. The temperature range is $-55 \sim 125$ centigrade, and the measured data is reliable and accurate. The humidity output of HS1101 is less affected by the temperature, the range is $1 \% \sim 99 \%$, and the response time is relatively short, which is about $5 \mathrm{~S}$, capable of meeting the needs of the system.

The processor module is the core part of sensor nodes, mainly responsible for the operation of the entire sensor nodes. It receives the measurement data from various types of sensors, and makes data processing, and then sends by the wireless communication module; it reads the data and control information received by the wireless communication module for the data processing, and realizes the control of the other modules. In addition, it determines when and where to send data and receives data from other nodes, and determines the next step of work state. Here we use TI's MSP430F149 ultra low power processor as the processor of the sensor node. It integrates a large number of peripheral modules into on-chip devices, which basically does not need to be extended to other peripheral. The kernel of MSP430F149 is 16bitRISC, and CPU clock frequency is $8 \mathrm{MHz}$. The capacity storage space is large, including 60kB FLASHROM and 2kB RAM [8]. There are 5 layers of sleep modes, the working voltage is $3.3 \mathrm{~V}$, and the power consumption is ultra low; with 12-bit AD converter and 2 serial communication interface, the function is complete and the integration degree is high, meeting the needs as wireless sensor network node processor.

The wireless communication module is mainly responsible for wireless communication with other sensor nodes, receiving and sending the collected information and exchanging the control information, generally including sending, receiving, idle and sleeping 4 working states. When it is in idle state, the node needs to listen to the wireless channel, and to detect whether there is data needed to receive; in sleep state, it is necessary to close the wireless communication module. This system uses CC1000 chip of Chipcon company as the wireless communication chip of sensor network node. The carrier frequency is $300 \sim 1000 \mathrm{MHz}$, with a low voltage $(2.1 \sim 3.6 \mathrm{v})$, low power consumption and high sensitivity, $-20 \sim-10 \mathrm{~dB}$ variable output power, and far transmission distance $(500 \sim 1000 \mathrm{~m})$, which is a kind of ideal UHF wireless communication chip [9]. 
Energy supply module is mainly responsible for providing the energy needed for the sensor node, which is the basic module of the wireless sensor network. The quality of design of power supply module will directly affect the lifetime, cost and volume of the sensor nodes. At present, the common power supply mode for sensor nodes is powered by batteries.

\subsection{WSN and RFID data integration model}

In general, the E-R model of WSN and RFID data is only for static data relation representation, which is difficult to meet the real-time requirements with high demand. But in the real world, we need more physical information to achieve full supervision of goods [6]. Therefore, it is necessary to establish a dynamic E-R data model which includes all directional information. We make a careful analysis of the entity set and the contact model in the simple E-R model of WSN and RFID data. It is found that the increase of state factor of time and information can reflect the dynamic changes of the E-R model. These factors include static time factors, static information factors, dynamic time factors, and dynamic information factors. Table 1 gives a detailed description of the four kinds of factors.

Based on the above contents, we built the E-R model of WSN and RFID integration data, as shown in Figure 9.

In the E-R data model shown in Figure 9, taking into account that the actual application does not need to record all the information changes in a certain period of time [Ts, Te], and only needs to determine whether the state change exceeds a defined range. Therefore, we use Dmin to record the minimum value of the information in the time period of [Ts,Te]. By Dmax, we mark the maximum value of the information in this period. We can dynamically update the Dmin and Dmax to determine whether the information is beyond the defined range [10]. By increasing the E-R data model of static time factor, static information factor, dynamic time factor and dynamic information factor, the system can not only greatly reduce the size of data records, but also can achieve efficient and dynamic database management. In order to more effectively monitor the logistics system status, the control layer needs the semantic processing of original data. Through data update, data filtering, data collection and other operations, we convert the data to the integrated data with a higher level, and store them in the above E-R model, to achieve efficient data management.

Table 1. Status factor for WSN integration with RFID data

\begin{tabular}{|l|l|}
\hline \multicolumn{1}{|c|}{ Status factor } & \multicolumn{1}{c|}{ Application object } \\
\hline Static time factor T & A time stamp that identifies when an event occurred \\
\hline Static information factor D & $\begin{array}{l}\text { A message flag, used to record incidental incidental information, such as } \\
\text { reader, sensor working status }\end{array}$ \\
\hline Dynamic time factor [Ts, Te] & $\begin{array}{l}\text { In a state of change in the relationship between the use of Ts and Te, } \\
\text { respectively, identify the beginning and end of the value of time, [Ts, Te] } \\
\text { recorded the time change }\end{array}$ \\
\hline $\begin{array}{l}\text { Dynamic information factor } \\
\text { Dmin, Dmax }\end{array}$ & $\begin{array}{l}\text { Dmin and Dmax respectively record the minimum and maximum infor- } \\
\text { mation of some information in [Ts, Te] period, and measure whether the } \\
\text { information is beyond the defined range by two values }\end{array}$ \\
\hline
\end{tabular}




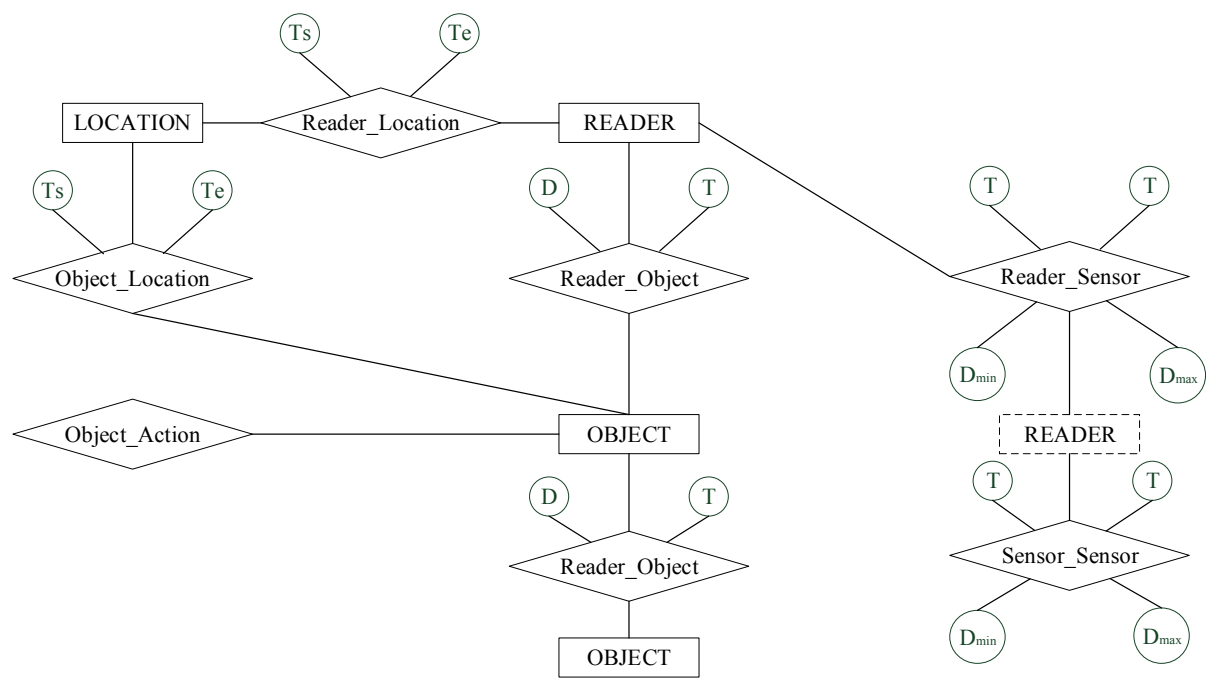

Fig. 9. E-R Model of WSN and RFID Fusion Data

\section{Conclusion}

Based on the wireless sensor network and RFID technology, according to the characteristics of airport logistics, a design scheme of monitoring system based on wireless sensor network and RFID is proposed. This paper introduces the overall structure and the working principle of the system, and specifically describes the division of functional modules and business process design. The system is applied to the airport logistics, which can achieve real-time tracking and positioning of airport cargo. The collection of goods relevant information is achieved during the transport, and realtime remote control is realized. As a result, the flow of goods can be effectively supervised, which improves the logistics information management and intelligent level, having high practical value and application prospect.

\section{References}

[1] Felemban, E. (2013). Advanced border intrusion detection and surveillance using wireless sensor network technology. International Journal of Communications, Network and System Sciences, 6(05), 251. https://doi.org/10.4236/ijens.2013.65028

[2] Zhong, N., Ma, J., Huang, R., Liu, J., Yao, Y., Zhang, Y., \& Chen, J. (2016). Research challenges and perspectives on Wisdom Web of Things (W2T). In Wisdom Web of Things (pp. 3-26). Springer International Publishing. https://doi.org/10.1007/978-3-319-4419861

[3] Kitsos, P. (Ed.). (2016). Security in RFID and sensor networks. CRC Press.

[4] Singh, A., Meshram, S., Gujar, T., \& Wankhede, P. R. (2016, December). Baggage tracing and handling system using RFID and IoT for airports. In Computing, Analytics and Securi- 
Paper-Application of Wireless Sensor Network and RFID Monitoring System in Airport Logistics

ty Trends (CAST), International Conference on (pp. 466-470). IEEE. https://doi.org/10.1109/CAST.2016.7915014

[5] Wang, B. (2014). RFID-based card reader design. In Applied Mechanics and Materials (Vol. 513, pp. 4035-4038). Trans Tech Publications. https://doi.org/10.4028/www.scien tific.net/AMM.513-517.4035

[6] Alyahya, S., Wang, Q., \& Bennett, N. (2016). Application and integration of an RFIDenabled warehousing management system-a feasibility study. Journal of Industrial Information Integration, 4, 15-25. https://doi.org/10.1016/j.jii.2016.08.001

[7] Bahrepour, M., Meratnia, N., \& Havinga, P. J. M. (2017). Automatic fire detection: a survey from wireless sensor network perspective. University of Twente Centre for Telematics \& Information Technology.

[8] Leu, J. S., Chiang, T. H., Yu, M. C., \& Su, K. W. (2015). Energy efficient clustering scheme for prolonging the lifetime of wireless sensor network with isolated nodes. IEEE Communications Letters, 19(2), 259-262. https://doi.org/10.1109/LCOMM.2014.2379715

[9] Magno, M., Polonelli, T., Benini, L., \& Popovici, E. (2015). A low cost, highly scalable wireless sensor network solution to achieve smart led light control for green buildings. IEEE Sensors Journal, 15(5), 2963-2973. https://doi.org/10.1109/JSEN.2014.2383996

[10] Al-Jemeli, M., \& Hussin, F. A. (2015). An energy efficient cross-layer network operation model for ieee 802.15.4-based mobile wireless sensor networks. Sensors Journal IEEE, 15(2), 684-692. https://doi.org/10.1109/JSEN.2014.2352041

\section{$7 \quad$ Author}

Le Wang is with Management College, Xi'an University of Architecture and Technology, Xi'an, Shaanxi, 710055, China.

Article submitted 03 December 2017. Published as resubmitted by the author 10 January 2018. 